\title{
RF-MEMS switches with AlN dielectric and their applications
}

\author{
MONTSERRAT FERNÁNDEZ-BOLAÑOS BADÍA ${ }^{1}$, PIERRE NICOLE ${ }^{2}$ AND ADRIAN MIHAI IONESCU ${ }^{1}$
}

\begin{abstract}
This paper reports on the potential of RF-MEMS technology based on aluminum nitride capacitive dielectric and nickelsuspended membranes to provide RF circuit functions in reconfigurable front-end radios. The RF performance of capacitive switches, distributed MEMS transmission lines (DMTLs) phase shifters for beam steering and tunable filters, including center frequency and bandwidth tuning of bandpass and band-stop filters are presented. Detailed characterization based on $\mathrm{S}$-parameter data demonstrates very promising figures of merit of all fabricated demonstrators from 5 to $40 \mathrm{GHz}$.
\end{abstract}

Keywords: RF MEMS switch, phase shifter, tunable filter, modelling, simulation and characterization of devices and systems Received 1 March 2011; Revised 30 April 2011; first published online 15 June 2011

\section{INTRDDUCTION}

In the last half-century, RF-MEMS switches have been extensively studied as they accomplish superior RF performance and higher linearity than semiconductor junction switches such as Positive-Intrinsic-Negative (PIN) diodes or Fieldeffect transistor (FETs) [1]. Despite their higher control voltages compared to some solid-state switches, electrostatic-actuated Micro-electro-mechanical-systems (MEMS) offer significant power savings as well as potential lower cost and the capability of miniaturization which result in attractiveness for their use in wireless applications.

In reconfigurable front-end RF ICs, functions like RF switching, filtering, and phase shifting in reflector array antennas for beam steering/forming networks (as illustrated in Fig. 1), can be realized with RF-MEMS components and systems using the same technology in a single fabrication process. RF MEMS offer new possibilities for improved performance and allow higher density and capacity of the overall systems.

In this paper we present capacitive shunt switches in terms of performance as well as their applications as phase shifters and tunable filters. In Section II, the state of the art of capacitive switches is studied and compared with different fabricated switch designs including those based on analog and digital capacitor concepts and switches with highly inductive components that were specifically designed as core devices for the applications presented in the subsequent sections. Then, in Section III analog and 2-bit digital true time delay (TTD) phase shifters are described as well as the co-integration of

\footnotetext{
${ }^{1}$ Ecole Polytechnique Fédérale de Lausanne (EPFL), Nanolab, Switzerland. Phone: +41216933973.

${ }^{2}$ THALES Airborne Systems, Elancourt, France.

Corresponding author:

M. Fernández-Bolaños Badía

Email: montserrat.fernandez-bolanos@epfl.ch
}

phase shifters and the dipole antennas for beam steering applications. Finally, tunable filters with different functionalities, as bandpass and band-stop filter configurations are shown in Section IV.

\section{RF-MEMS CAPACITIVE SWITCH - SWITCHING}

\section{A) Classification}

Capacitive switches are mainly divided into two subcategories depending on their different functionalities; the RF-MEMS capacitive switch or analog MEMS capacitor, with high isolation and an increased capacitance ratio, $\mathrm{Cr}$ $(>30)$ and the MEMS switch capacitor or digital MEMS capacitor with a moderate and usually defined $\mathrm{Cr}<5$. Analog MEMS capacitors can be used for signal routing purposes, in reconfigurable front-ends, digitized capacitor banks, and reconfigurable antenna orientation or apertures. In order to achieve high isolation, many different configurations have been reported based on shunt and series switches [1-8], low spring constant anchoring [3], inductive behavior [8], and/or using high dielectric constant materials $[4,6,7]$.

On the other hand, the digital MEMS capacitor is generally used as part of tunable systems or reconfigurable networks, for example filters, phase shifters, or impedance matching with two known capacitive states. The concept of the digital capacitor was first introduced by Hayden and Rebeiz [9] and is defined as the standard MEMS capacitive switch in series with a fixed small capacitance possibly realized with metalinsulator-metal (MIM) capacitor [10], metal-air-metal (MAM) capacitor [11], or interdigitated capacitors. Figure 2 illustrates for comparison purposes the capacitance versus voltage curve ( $\mathrm{CV}$-curve) of a standard analog capacitor and 


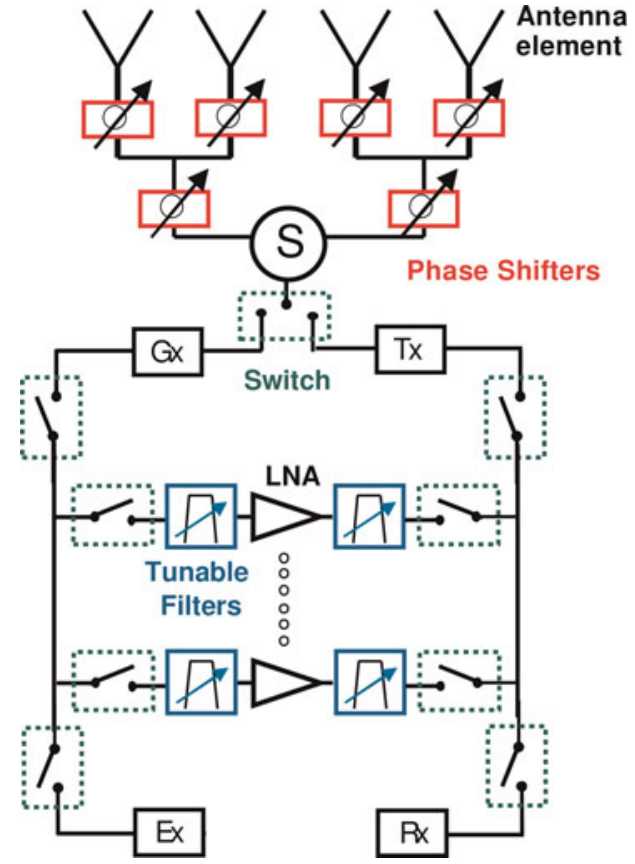

Fig. 1. Reconfigurable front-end RF IC typical simplified architecture.

a digital capacitor made with MIM fix series capacitances. The high capacitance ratio of the fabricated analog capacitor $(\mathrm{Cr}=$ 20-40) and the MIM digital capacitor designed can be observed to have a $\mathrm{Cr}=3$.

\section{B) State of the art}

Capacitive shunt switches have been realized with a large variety of dielectrics. Silicon nitride $\left(\mathrm{Si}_{3} \mathrm{~N}_{4}\right)$ with a dielectric constant of $\varepsilon_{r}=7.5$ is the most popular material for capacitive MEMS [2, 3, 8]; however, other high dielectric constant materials such as strontium titanate oxide $\left(\varepsilon_{r}=120,[4]\right)$, piezoelectric lead zirconate titanate $\left(\varepsilon_{r}=190,[6]\right)$, aluminum nitride (AlN, $\varepsilon_{r}=9: 8,[7]$ ), and even dielectric-less structures [5] present excellent RF performance up to $40 \mathrm{GHz}$. All of them have demonstrated to achieve very low insertion losses $(<0.2 \mathrm{~dB}$ at $40 \mathrm{GHz})$ and high isolation $(>30 \mathrm{~dB})$ with an elevated capacitance ratio $(\mathrm{Cr}>30)$ and acceptable switching

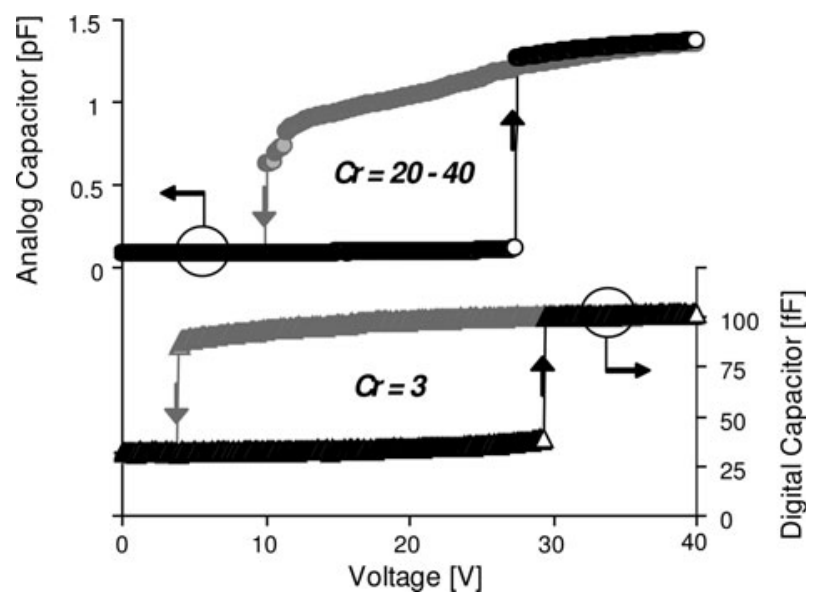

Fig. 2. $C V$-curves of an analog capacitor and an MIM digital MEMS capacitor. time $(3-20 \mu \mathrm{s})$ at a relatively low actuation voltage $(<30 \mathrm{~V})$ for billions of cycles $[6,7,12,13]$.

The RF-MEMS capacitive shunt switches as well as the other MEMS presented in this article use a common fabrication process developed at the Fraunhofer Institute of Silicon Technology (ISiT). The original process includes a combination of thin and thick nickel (Ni) for the suspended membrane and the anchors (and/or stiffening bars), respectively. In addition, AlN is used as a high-lifetime dielectric. The fabricated capacitive switches have performances comparable to state of the art of the technology.

Figure 3 compares the most representative RF capacitive switches regarding the area and the isolation with respect to the applied actuation voltage. In the figure, the selected dark squares represent the desired performances of a switch. The fabricated capacitive switch is in general more compact and achieves higher isolation than their counterpart MEMS switches [1-6], for similar actuation voltages (Fig. 3(a)). In addition, Fig. 3(b) depicts the capacitance ratio $(\mathrm{Cr})$ for each switch, which is in principle a good figure of merit of the $\mathrm{RF}$ capacitive switch performance. However, a device with a very high capacitance ratio $(\mathrm{Cr} \sim 600,[4])$ could result in poor isolation $(<20 \mathrm{~dB})$ due to bad dielectric RF properties.

It is noteworthy to remark in Fig. 4 that a trade-off exists between low spring constants (for low actuation voltages purpose [3]) and the switching time or speed of the switch following a linear dependency. A known way of reducing the switching time is by device miniaturization [13]. The price to pay is a decrease in the RF performance.

The superior characteristics of the fabricated RF-MEMS switch can be attributed to excellent mechanical behavior of $\mathrm{Ni}$ membranes, good anchoring designs that reduce the effect of induced stress, and good AlN dielectric properties.

\section{C) Design and fabrication}

The RF-MEMS capacitive shunt switches have been developed at ISiT as part of a multi-project wafer consisting of an eightmask process fabricated on highly resistive Si substrate $\left(>_{3} \Omega\right.$ $\mathrm{cm})$, presented in detail elsewhere $[14,15]$. The devices are loaded on a $78 \Omega$ high-impedance line consisting of $2 \mu \mathrm{m}$ insulated thermal oxide and $3 \mu \mathrm{m}$ gold ( $\mathrm{Au}$ ) CPW. The total CPW width is $300 \mu \mathrm{m}$ with S/W/S of $125 / 50 / 125 \mu \mathrm{m}$. The process combines to achieve a good mechanical and reliability behavior; a thin $1 \mu \mathrm{m}$ evaporated $\mathrm{Au} / \mathrm{Ni} / \mathrm{Au}$ multimetal layer for the movable membrane, a thick $14 \mu \mathrm{m}$ electroplated $\mathrm{Ni}$ for the anchors and stiffening bars and $300 \mathrm{~nm}$ medium-dielectric constant amorphous AlN dielectric. The release structure presents an approximate air gap of $2 \mu \mathrm{m}$. Figure 5 illustrates a cross-sectional view of a capacitive MEMS switch with a fixed-fixed beam configuration showing the main layers of the process and a detailed SEM (scanning electron microscopy) image of a fabricated device.

Different analog and digital RF-MEMS capacitive shunt switches have been specifically designed using mainly three kinds of low spring constant anchoring (spring type, meander type and crabbed-meander type) to achieve different actuation voltages as well as specific inductive components. Each of them was conceived to be used as core devices for applications in phase shifters and tunable filters and their design was optimized for their work at specific frequencies. Figure 6 depicts SEM images of the main switches with 


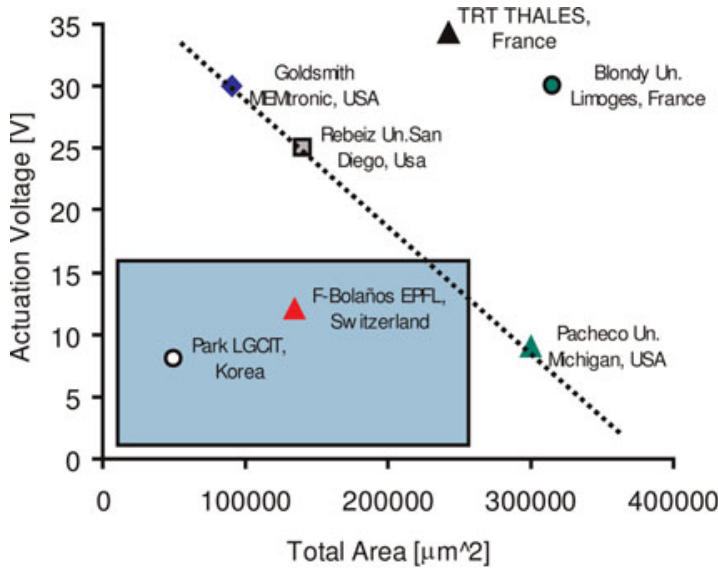

(a)

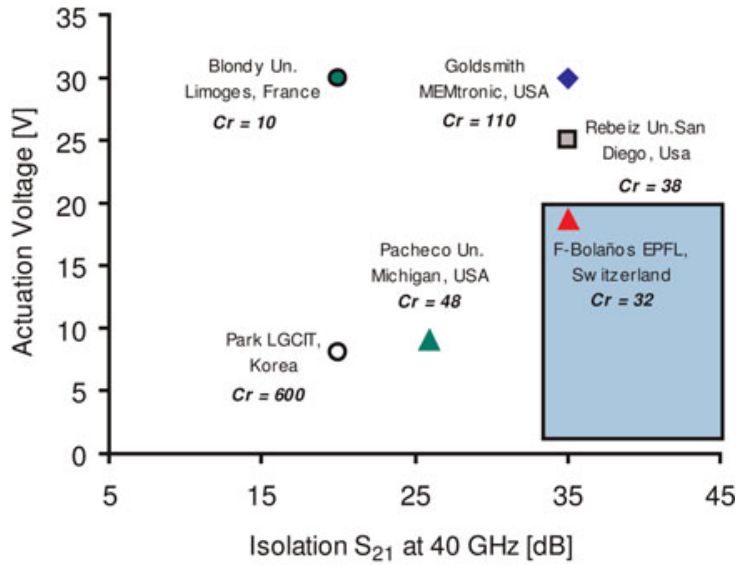

(b)

Fig. 3. Comparison of the actuation voltage of state-of-the-art switches versus (a) the total switch area and (b) the isolation, $\mathrm{S}_{21}$, at $40 \mathrm{GHz}$ as well as the $\mathrm{Cr}$.

different anchoring indicating the application for which they were designed.

\section{D) Characterization and modeling}

The switch is electrostatically actuated directly from the bottom electrode by applying a DC voltage inline with the $\mathrm{RF}$ signal of the CPW central conductor. The suspended bridge (top electrode of the capacitor) is anchored to the ground plane of the CPW (Fig. 7) and is actuated, thanks to the resulting electrostatic force.

Device characterization from 6 to $40 \mathrm{GHz}$ is carried out on E8361A Vector Network Analyzer and the calibration is performed using an external short-open-line-thru commercial calibration kit. De-embedding corrections are performed by measuring a thru line structure in the wafer to remove the losses and the parasitic pad capacitances of the unloaded line.
A $12 \mathrm{~V}$ low actuation RF-MEMS shunt capacitive switch is designed using crabbed-meander-type anchoring (shown in Fig. 6(b)) with an approximate spring constant $\left(K_{S}\right)$ of $9 \mathrm{~N} / \mathrm{m}$. The achieved capacitance ratio, $\mathrm{Cr}$, is 27 at pull-in voltage $\left(V_{P I}\right)$, while it increases up to 40 with a higher actuation voltage of $40 \mathrm{~V}$. Figure 8 presents the $S$-parameter measurements of the shunt switch. The device provides good RF performances with isolation better than $37 \mathrm{~dB}$, an insertion loss of $0.2 \mathrm{~dB}$ and a return loss of $12 \mathrm{~dB}$ at $40 \mathrm{GHz}$. In addition, reliability tests have been performed in hot switching conditions $\left(+5 \mathrm{dBm}\right.$ RF power) over $2 \times 10^{9}$ cycles without permanent failure or stiction [7] and an excellent thermal behavior has been measured from 100 to $-130^{\circ} \mathrm{C}$ with a $V_{P I}$ shift less than $2 \mathrm{~V}$ for the entire temperature range, $\Delta T=230^{\circ} \mathrm{C}[10]$.

The shunt switch is modeled by an accurate equivalent circuit T-model as shown in Fig. 9. Where, $C_{t}, L_{t}$, and $R_{t}$ correspond to the unloaded CPW and their values for a $460 \mu \mathrm{m}$

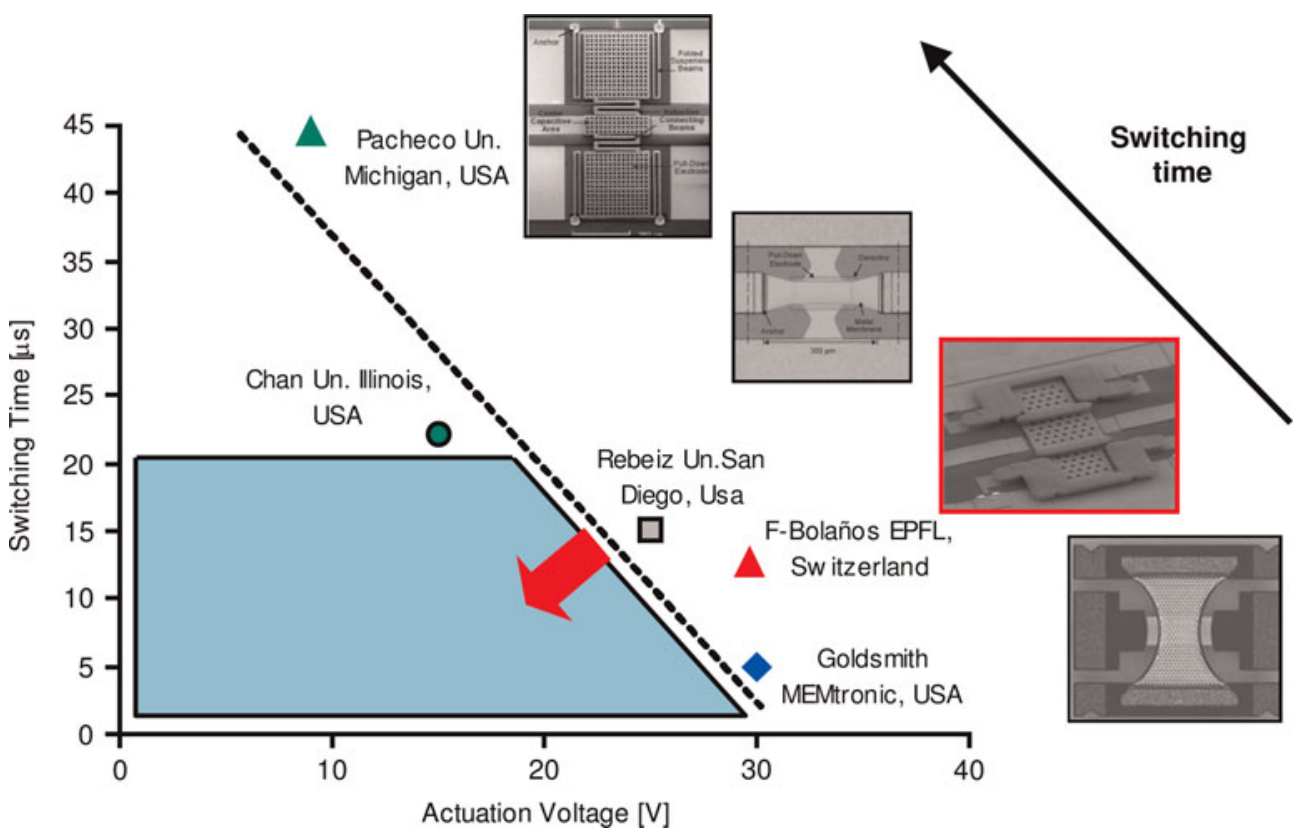

Fig. 4. Comparison of the switching time of the state-of-the-art switches versus the actuation voltage. 


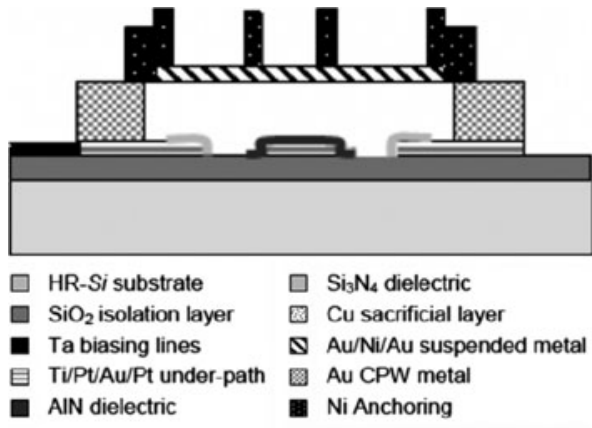

(a)

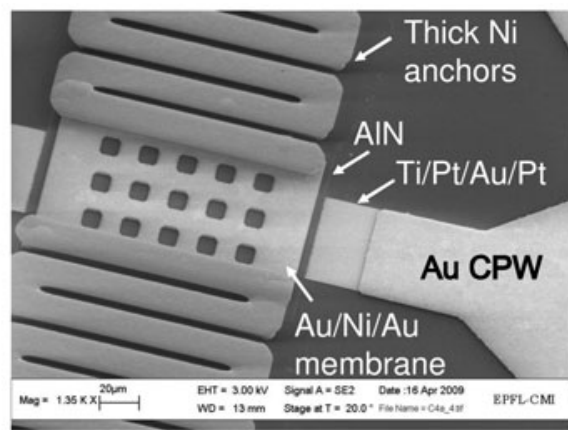

(b)

Fig. 5. (a) Cross-sectional view and (b) SEM image of a detail in a fabricated device showing the different layers of the process.

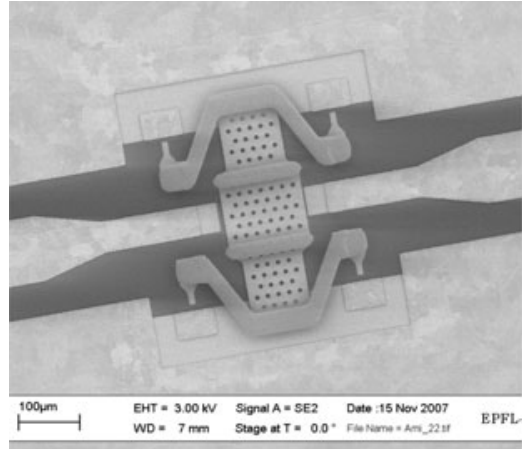

(a)

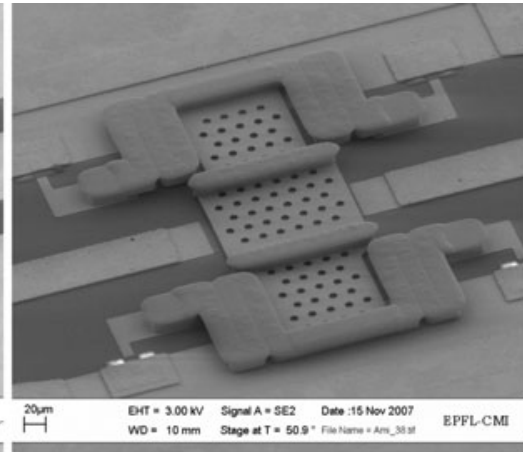

(b)

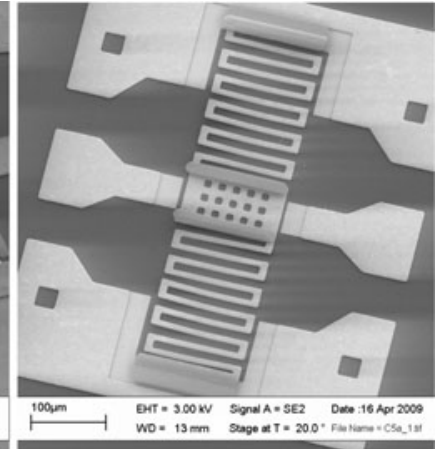

(c)

Fig. 6. Three different capacitive switches designs used as core devices for the following applications: (a) spring type and (b) crabbed meander type used in combination for analog and digital phase shifter and bandpass filters and (c) standard meander-type for stop band filter applications.

length $\left(d_{\text {ref }}\right)$ are: $C_{t}=35 \mathrm{fF}, L_{t}=143 \mathrm{pH}$, and $R_{t}=0.1 \Omega$ (extracted from the thru de-embedded line). $C_{M E M S}, L_{p}$, and $R_{p}$ are the components of the parallel shunt impedance $(Z p)$ and correspond to the MEMS membrane. When the switch is not actuated, $C_{M E M S} U_{p}$ is very small and $L_{p}$ and $R_{p}$ can be neglected. Finally, $\Delta L_{s}$ and $\Delta R_{s}$ are corrective series elements used to keep the length of the discontinuity close to zero when modeling the device. As a result, $\Delta L_{s}$ can be negative while $\Delta R_{s}$ is expected to be positive [16]. MEMS switch circuit parameters are shown in Table 1 and have been extracted from the $S$-parameter measurements of the crabbed-meander-type switch and using the equivalent T-circuit model (Fig. 9).

Agilent-Advanced Device System (ADS) software has been used to perform equivalent circuit simulations of the extracted parameters and results are plotted in Fig. 10(a). Moreover, $3 \mathrm{D}$ finite-element electromagnetic (EM) full-wave simulations of the crabbed-meander-type capacitive switch are performed by means of Ansoft's HFSS software and depicted in Fig. 10(b). Both simulations are in good agreement with the measurements validating the extracting parameter method and the HFSS ${ }_{3} \mathrm{D}$ EM model.

\section{E) Capacitive switches with a significant inductive component}

In order to achieve higher isolation at specific desired frequencies, one possible solution is to design a switch with a highly dominant inductance component. A highly inductive design can be achieved by varying the anchoring or the switch design over the slots of the CPW as in the case of the meandertype switch (shown in Fig. 6(c)) However, in this kind of design high isolation is only obtained in general in a relatively narrow bandwidth.

It can be observed in Fig. 11 that a minimum of $5 \mathrm{~dB}$ improvement in isolation can be achieved compared to the standard crabbed-meander-type anchoring capacitive switch previously presented (Fig. 8). The membrane bridge inductance has been varied from 10 to $324 \mathrm{pH}$ in five different configurations. They result in down-state natural resonant frequencies, $f_{\mathrm{o}}$ (or isolation region) at $7.8,13.3,17.8$, and $27.5 \mathrm{GHz}$ and higher than $40 \mathrm{GHz}$ for the standard switch. A lower than $0.2 \mathrm{~dB}$ insertion loss is achieved for all devices

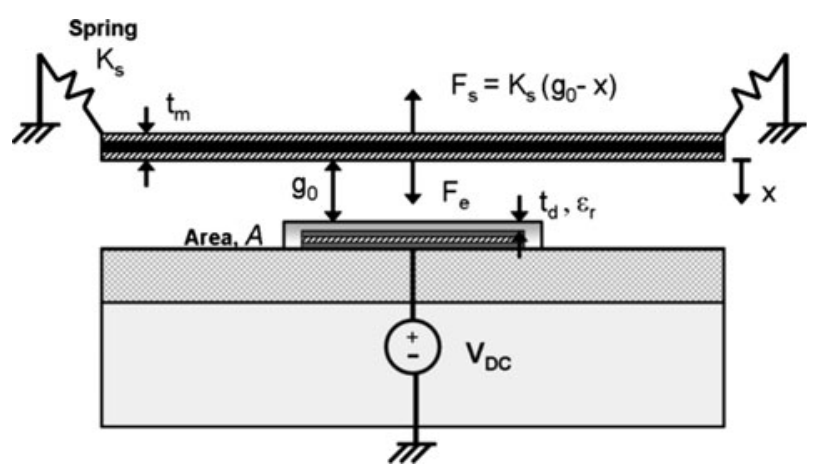

Fig. 7. Schematic view of a general RF-MEMS capacitive shunt switch electrostatically actuated. 


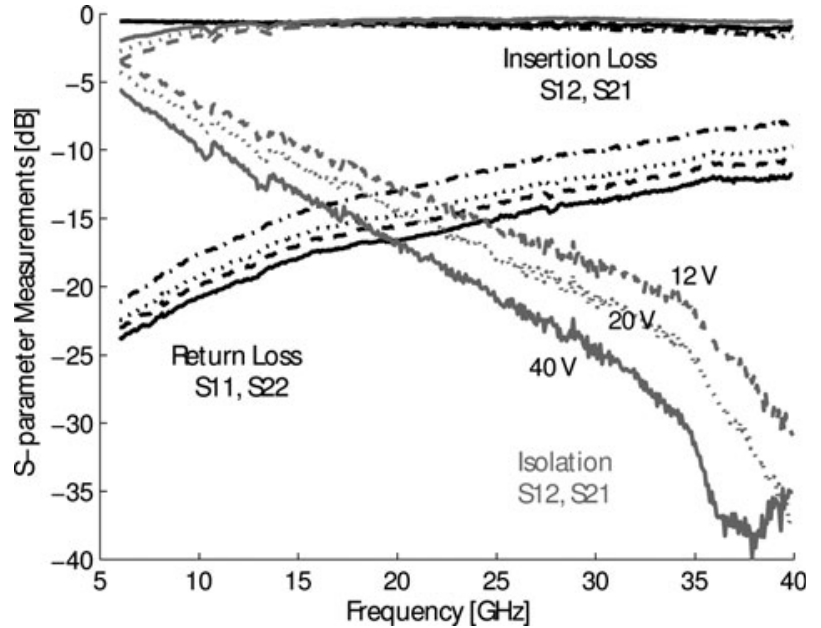

Fig. 8. $S$-parameter measurements of crabbed-meander-type anchoring capacitive shunt switch (shown in Fig. 6(b)) for different applied voltages. The pull-in voltage, $V_{P I}$, occurs at $12 \mathrm{~V}$.

with a minimum isolation of $12 \mathrm{~dB}$ in the lower frequency band (at $7.8 \mathrm{GHz}$ ). Insertion loss and isolation measurements $\left(S_{21}\right)$ of a set of inductive switches in the up and down state, respectively, are depicted in Fig. 11.

\section{F) Digital MEMS capacitor design}

The series fixed capacitance, $C_{F I X}$, is designed with a value much smaller than $C_{M E M S}$ DOWN to limit the value of the total down-state digital MEMS capacitance. The idea consists in creating two well-defined capacitive states less dependent on the technology. This device eliminates the uncertainty of the MEMS down-state capacitance, which is much dependent on the fabrication process (due to the roughness and the planarization process of the top and bottom electrodes) and it is very difficult to accurately estimate its value in advance ( $>50 \%$ standard deviation). The total contribution of the MEMS and fixed capacitances placed in series configuration results to be similar to the smallest capacitance value, $C_{F I X}$. Consequently, small or medium changes in the value of the

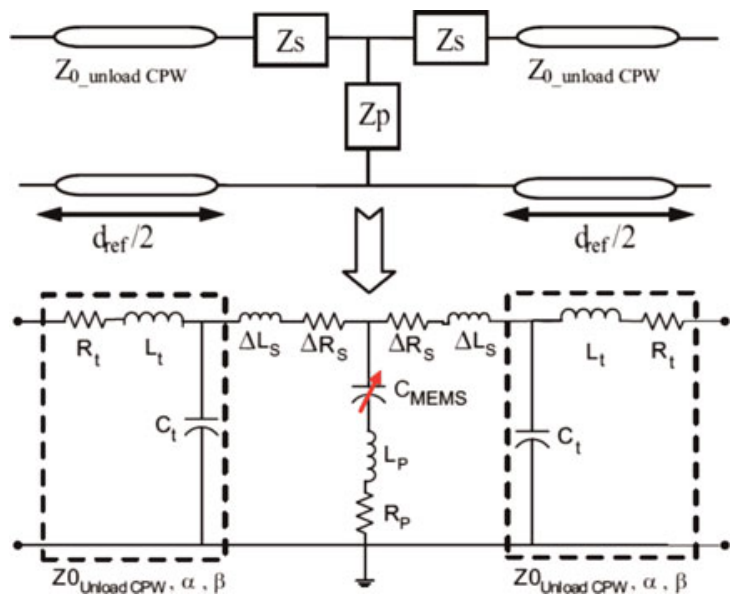

Fig. 9. Equivalent T-model used to extract the circuit parameters of the switch from RF measurements.
$C_{\text {MEMS DOWN }}(1-1.5 \mathrm{pF}$, see Table 1$)$ can be neglected for the device performance.

Among different $C_{F I X}$ approaches considered to design digital MEMS capacitors, interdigitated capacitors seem to be the least dependent on the technology. The capacitance is realized in a single metal photolithography step and the accuracy of its value depends only on the lateral resolution of the equipment. However, it is not a miniaturized solution, overall if the interdigitated metal layer (in our case $\sim 400 \mathrm{~nm} \mathrm{Ti} / \mathrm{Pt}$ / $\mathrm{Au} / \mathrm{Pt}$ underpath metal) is relatively thin. MAM capacitors mainly depend on the sacrificial layer (which will define the air gap thickness) and on their uniformity around the wafer. Unfortunately, the air gap is highly dependent on the technology and usually has a minimum of $25 \%$ uncertainty. Finally, MIM capacitors depend only on the lateral resolution of photolithography and on the dielectric thickness. The dielectric layer can be deposited with high precision and its thickness can differ by a very few percentage of error. In addition, MIM capacitors are very compact and result in the most interesting approach [10].

Figure 2 illustrates the capacitance versus voltage of a standard analog capacitor and an MIM digital capacitor. It can be appreciated how MIM digital capacitors are almost independent on the technology. The total contribution of the up-/ down-state capacitances are: $C_{\text {Total UP }}=33.5 \mathrm{fF}$ and $C_{\text {Total }}$ DOWN $=99 \mathrm{fF}$ which corresponds almost exactly to the designed $\mathrm{Cr}$ of 3 [10]. The designed $C_{F I X}$ results in a value of $90 \mathrm{fF}$. Unfortunately, due to this added series fixed capacitance the actuation voltage needed to snap down the MEMS membrane will substantially increase by an approximate factor of 1.3-1.5.

Figure 12 depicts the $S_{21}$ phase or argument of the individual digital switch (of $0.5 \mathrm{~mm}$ cell length). The figure shows a phase shift between both states of $15^{\circ}$ at $17 \mathrm{GHz}$. These digital capacitors will be used as the core device for phase-shifting applications in the next section. For a phase shift of $180^{\circ}$ at $17 \mathrm{GHz}$, approximately 13 switches periodically loaded in a distributed MEMS transmission line (DMTL) CPW of $6 \mathrm{~mm}$ total length would be needed.

\section{RF-MEMS TRUE-TIMEDELAY (TTD) LINE - PHASE SHIFTING}

RF-MEMS capacitors have been widely investigated for their application in DTMLs phase shifters, both in analog [17] and digital modes $[1,9-11]$. The idea is to periodically load a coplanar waveguide (CPW) with voltage-controlled varactors (or two-state digital capacitors [10]), to tune the distributed capacitance, the phase velocity and the propagation delay in the line. By actuating the loaded MEMS capacitors, a different phase state can be achieved. The main advantage of DMTL phase shifter is that it behaves as a real TTD line, with a constant time delay over a wide-band of frequencies. Electronically beam-steered antenna consists of dynamically switching the beam configurations of an antenna array by controlling the relative phases at the input of each element to change the orientation of the whole antenna beam [17].

Figure 13 depicts the typical $C-V$ curve of both, a single digital capacitor and a full array of capacitors loaded on a CPW, namely the DMTL phase shifter, which are simultaneously operated. One can observe a quasi-identical pull-in voltage $\left(V_{P I}\right)$ for all the switches $\left(\sim_{22} \mathrm{~V}\right)$ and a pull-out 
Table 1. T-model circuit parameter extraction from $S$-parameter measurements.

\begin{tabular}{|c|c|c|c|c|c|c|c|}
\hline \multirow[t]{2}{*}{ Parameter at $6-40 \mathrm{GHz}$} & \multicolumn{7}{|c|}{ Meander-type capacitive shunt switch } \\
\hline & Up & & & & Down & & \\
\hline Voltage & o $V$ & $8 \mathrm{~V}$ & $10 \mathrm{~V}$ & $12 \mathrm{~V}$ & $13 \mathrm{~V}$ & $20 \mathrm{~V}$ & $40 \mathrm{~V}$ \\
\hline \multicolumn{8}{|l|}{ Parallel $Z_{p}$} \\
\hline$C_{p}$ & $40 \mathrm{fF}$ & $44.5 \mathrm{fF}$ & $48.5 \mathrm{fF}$ & $59 \mathrm{fF}$ & $1.11 \mathrm{pF}$ & $1.28 \mathrm{pF}$ & $1.55 \mathrm{pF}$ \\
\hline$R_{p}$ & - & - & - & - & & $0.25 \Omega$ & \\
\hline$L_{p}^{p}$ & - & - & - & - & & $12 \mathrm{pH}$ & \\
\hline \multicolumn{8}{|l|}{ Series $Z_{s}$} \\
\hline$\Delta L_{s}$ & $-10 \mathrm{pH}$ & & & & $-10 \mathrm{pH}$ & & \\
\hline$\Delta R_{s}$ & $2.64 \Omega$ & & & & $0.54 \Omega$ & & \\
\hline Natural frequency & - & - & - & - & $43.6 \mathrm{GHz}$ & $40.6 \mathrm{GHz}$ & $36.9 \mathrm{GHz}$ \\
\hline
\end{tabular}

voltage $\left(V_{P O}\right)$ slightly higher than $10 \mathrm{~V}$. The polarity of the applied voltage should not have any influence on the actuation since the electrostatic force is proportional to the voltage squared. A symmetrical curve around $V=0$ is achieved, which suggests either that no mobile residual charges exist or that they are compensated.

\section{A) Analog DMTL phase shifter}

Distributed MEMS TTD phase shifters with an analog control [18] are limited by the MEMS intrinsic instability appearing at a third of the original air gap. This corresponds to a voltagecontrollable capacitance ratio $(\mathrm{Cr})$ that is lower than 1.5 during the stable up-state. Analog DMTL phase shifters are actuated below the pull-in voltage of the MEMS switches achieving a continuous and quasi-linear phase shifting. Once the membranes snap down, the $\mathrm{Cr}$ can rise up to 20100. Such high $\mathrm{Cr}$ is usually advantageous for switching application where high isolation is needed. However, for other applications such as TTD phase shifters, even if it might seem advantageous for miniaturization purposes, the mismatch produced by such an enormous $\mathrm{Cr}$ is prohibitive.

The actuation is similar to that of the individual switches. DC voltage is applied through the central conductor of the CPW inline with the RF signal. Figure 14 illustrates the fabricated analog DMTL phase shifter using crabbed-meander-type switches.

The main advantage of analog DMTL is its continuous phase shifting with a single voltage command. However, this phase shifter approach is not very compact and the maximum phase shift is limited to $\mathrm{Cr}<1.5$. The analog phase shift achieves a maximum continuous shifting of 4.36 ps time delay $(\Delta D)$, in a wide frequency band (from 5 to $25 \mathrm{GHz}$ ). The phase shifter includes 13 crabbed-meander-type anchoring analog capacitors periodically loaded in a CPW transmission line and separated by $400 \mu \mathrm{m}$ from center to center of each individual MEM cell. The total size of the phase shifter is $5.4 \times 0.6 \mathrm{~mm}^{2}$.

The insertion loss is on average $2.39 \mathrm{~dB}$ at $17 \mathrm{GHz}$ and the return loss is better than $15 \mathrm{~dB}$ for all the states over the entire frequency range as shown in Fig. 15.

The analog DMTL phase shifter results in a moderate phase shift of $53 \% \mathrm{~cm}$ and $6 \% \mathrm{~dB}$ at $17 \mathrm{GHz}$. The linearity of the differential phase shift with respect to frequency can be observed up to $25 \mathrm{GHz}$.

\section{B) Digital DMTL phase shifter}

In order to achieve more controllable and miniaturized solutions, digital phase shifters are adopted using periodically loaded digital capacitors with MIM fixed capacitors and are designed to have a higher capacitive ratio $(\mathrm{Cr} \sim 3)$ than their analog counterparts. Digital DMTLs result in a larger amount of degrees per centimeter performance than the analog phase shifters, thanks to its greater design compactness related to the higher $\mathrm{Cr}$. However, they are limited to discrete states.

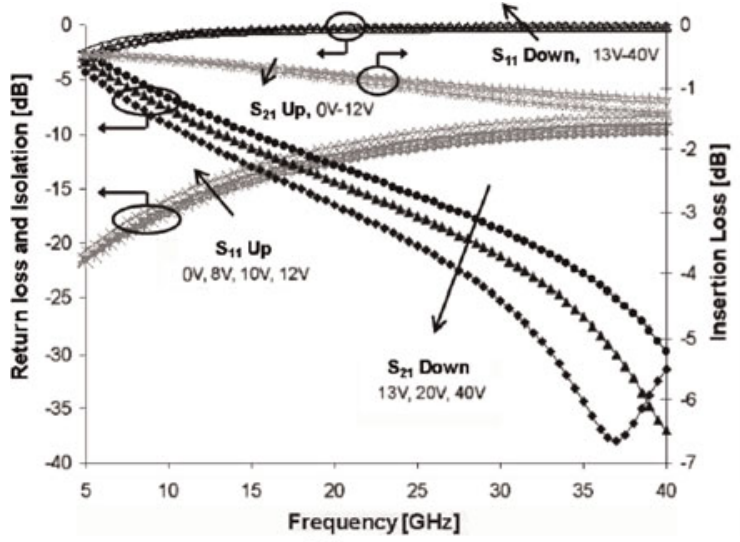

(a)

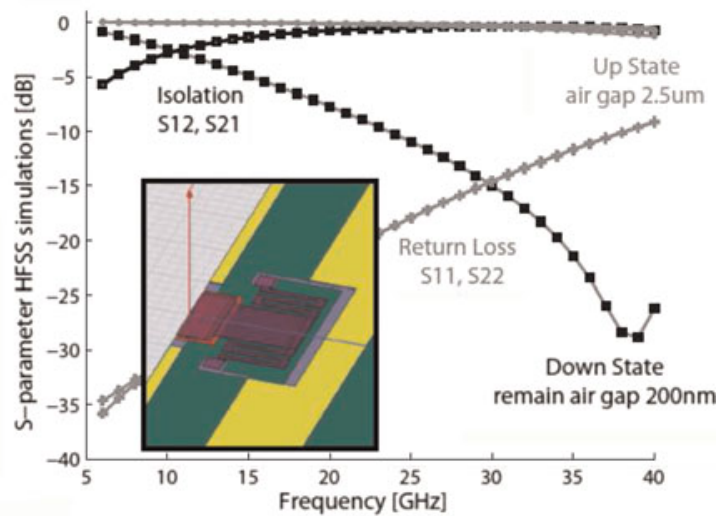

(b)

Fig. 1o. (a) ADS circuit T-model simulations for all the actuation voltages shown in Table 1. (b) S-parameters' HFSS Ansoft simulation of the $3 \mathrm{D}$ EM model of the crabbed-meander-type anchoring capacitive switch in the up and down states. 


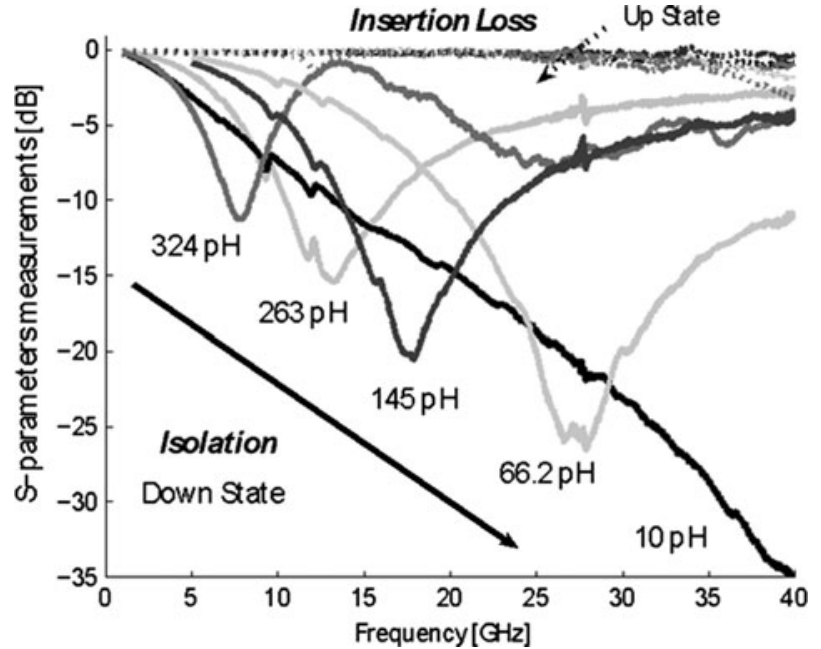

Fig. 11. Insertion loss and isolation measurements, $\left(S_{21}\right)$ of the crabbed-meander-type anchoring switch (black line) and highly inductive capacitive shunt switches made specifically to resonate at a certain frequency.

In principle, with a single control voltage, just two phase states are possible in 1-bit DMTL line $[7,10,11]$. In order to achieve multi-state phase shifters, the standard solution is to add multi-bit control commands, which substantially increase the complexity of the device [9, 19]. However, other approaches could be envisioned. For instance, a three-state digital phase shifter consisting in switches with different spring constant devices would result in switches with different pull-in voltages. Such a phase shifter could achieve at least three phase shifters states with a single actuation command. This seems to be a superior solution in terms of simplicity and miniaturization for phase shifters when only three discrete states are needed.

However, multi-bit actuation phase shifters remain the standard design method by cascading a number $N$ of DMTLs (cascaded-bit, CB-DMTL). Each section provides a delay corresponding to a bit weight (related to the number of switches and corresponding length) [19] and are independently actuated by $N$ voltage controls. This kind of phase shifter suffers from poor matching in the intermediate

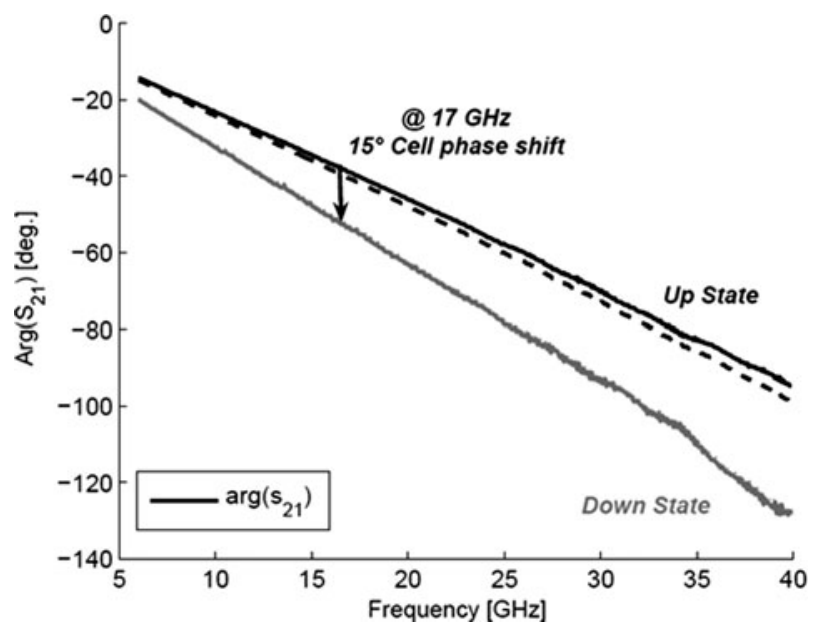

Fig. 12. Measurements of the $S_{21}$ argument for the up/down states of the digital MEMS capacitor. It represents the phase shift achieved for an individual cell when switching from both states.

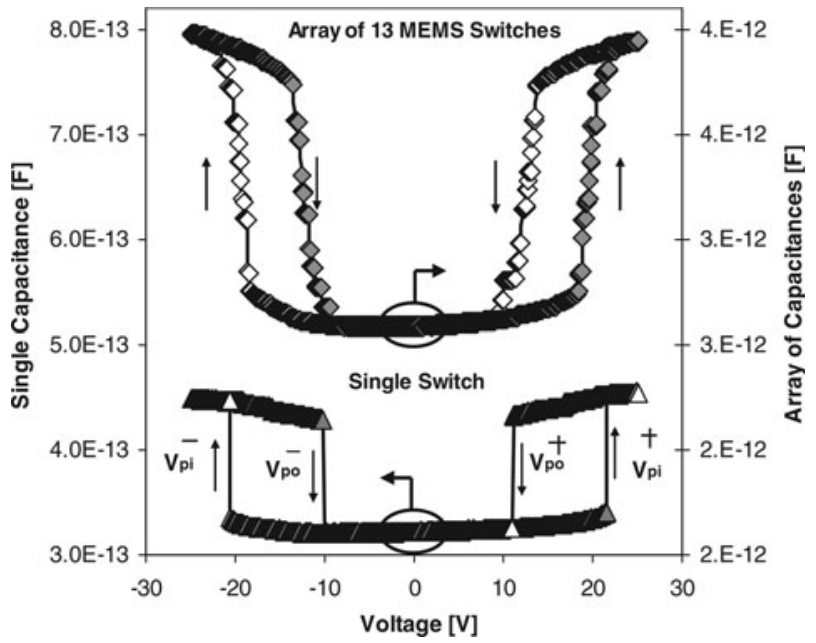

Fig. 13. Measured $C-V$ curves of a single digital capacitor and the array of parallel-loaded 13 MEMS capacitors forming the phase shifter with approximately $22 \mathrm{~V}$ pull-in for all the 13 MEMS capacitors simultaneously operated.

states. On the other hand, interlaced-bit configuration (IB-DMTL) has demonstrated theoretically to alleviate this problem [20]. The price to pay is a higher complexity of the optimization design, overall if more than 2 bit are required.

A two-bit DMTL phase shifter has been designed with IB-DMTL configuration and optimized to attain minimum mismatch at $17 \mathrm{GHz}$ (frequency of interest for the beam steering application) in all its phase states. An SEM image of the fabricated phase shifter is shown in Fig. 16. Eighteen springtype anchoring bridges (with 8 and 10 bridges for bit $A$ and bit $B$, respectively) are loaded interlaced and spaced by $344 \mu \mathrm{m}$. The total size of the 2-bit IB-DMTL phase shifter is $6.1 \times 0.9 \mathrm{~mm}^{2}$. The bits are individually actuated using highresistivity bias lines made of $25 \mathrm{~nm}$ of tantalum (100 $\Omega / \mathrm{sq}$ ) to prevent the coupling between the RF signals and the bias circuitry.

The fabricated 2-bit IB-DMTL phase shifter achieved a de-embedded insertion loss of $1.9 \mathrm{~dB}$ on average and an excellent optimized matching better than $21 \mathrm{~dB}$ at $17 \mathrm{GHz}$ for all the phase shifter states (Fig. 17(a)). In addition, an acceptable return loss (better than $12 \mathrm{~dB}$ ) is obtained from 5 to $25 \mathrm{GHz}$.

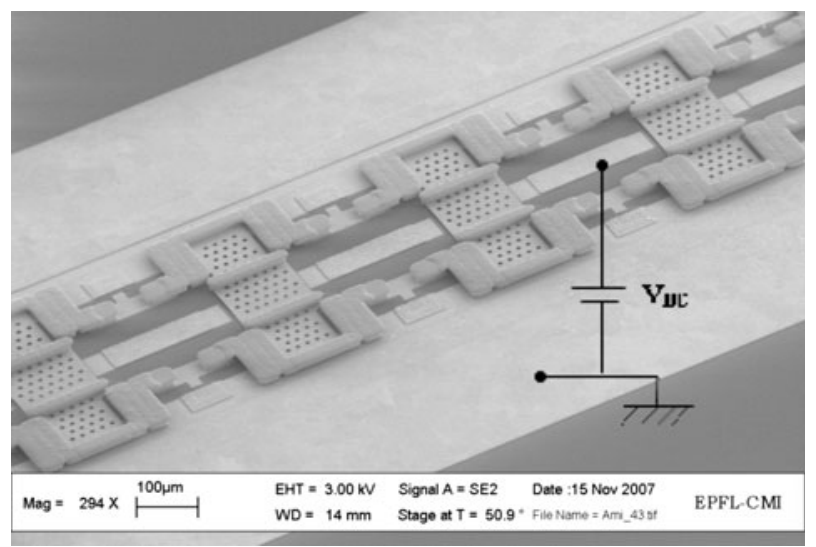

Fig. 14. SEM image of the fabricated distributed MEMS analog phase shifter using 13-loaded RF-MEMS analog capacitive switches which corresponds to the one measured in Fig. 8 (only four switches are shown in this figure). 


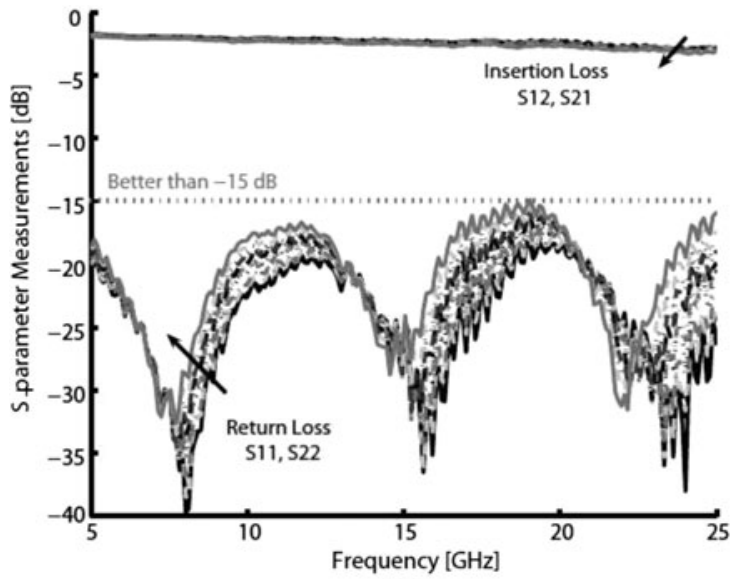

(a)

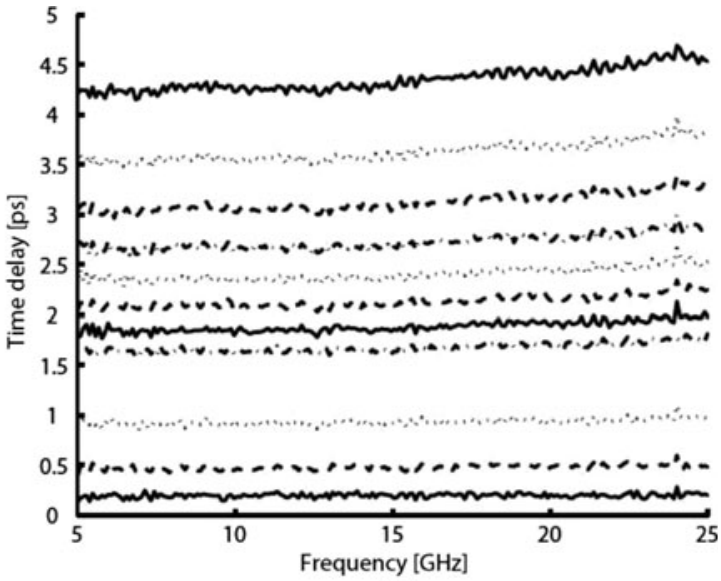

(b)

Fig. 15. (a) S-parameter measurements and (b) continuous TTD of the analog DMTL phase shifter based on analog MEM capacitors.

Constant and equidistant weight-bit time delays of 6.2, 11.9, and $18 \mathrm{ps}$, which correspond to 01,10 , and 11 states, respectively, were measured (Fig. 17(b)).

The loaded characteristic impedance of the phase shifter in the different states is shown in Fig. 18(a) up to $40 \mathrm{GHz}$. The real impedance corresponds to $54,50.9,48.1$, and $45.4 \Omega$ from 00 to 11 phase states. These values demonstrate and successfully validate the IB-DMTL configuration proposed in [20] as well as the frequency matching optimization method. The figures of merit of the phase shifter result in $180 \% \mathrm{~cm}$ and $20 \% \mathrm{~dB}$ (without de-embedding the structure) at $17 \mathrm{GHz}$ as shown in Fig. 18(b).

\section{C) $17 \mathrm{GHz}$ fully integrated antenna beam steering concept}

A full integration of an antenna beam steering concept has been performed in a single chip for wireless sensor nodes. The system includes a dipole antenna, a balun, and a 2-bit digital DMTL phase shifter. An SEM of the entire fabricated system is depicted in Fig. 19(a). The dipole antenna is patterned in a single thick metal as the CPW lines as well as

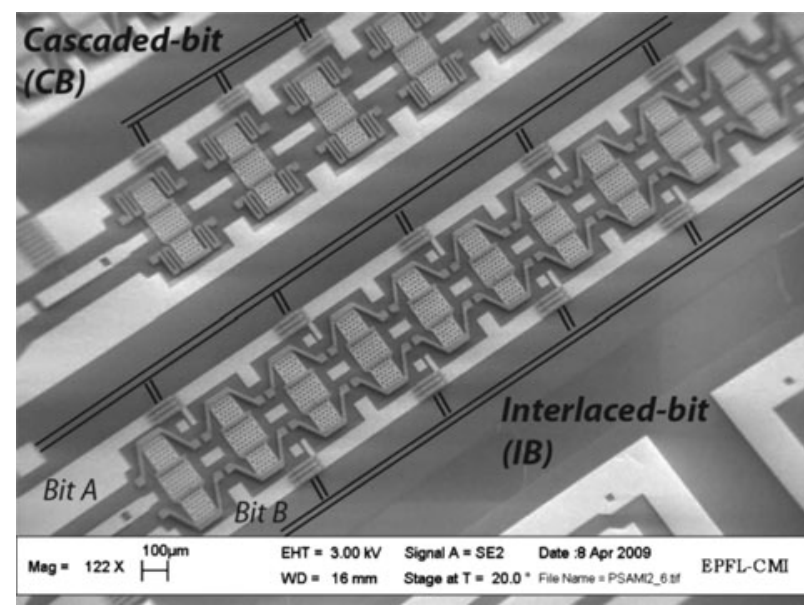

Fig. 16. SEM image of 2-bit DMTL phase shifters in interlaced (IB) and cascaded (CB) bit configuration loaded in a CPW and actuated through high-resistivity bias lines. the balun but with two fixed air bridges to connect both ground planes of the CPW.

The antenna and the phase shifter designs were optimized to have good matching and return loss $(>40 \mathrm{~dB})$ at the desired RF frequency $(17.2 \mathrm{GHz})$ using a surface-passivated HR-Si substrate [21] which was thinned to $300 \mu \mathrm{m}$ to improve the radiation pattern performance. The balun provides a right-angle transition from the $\mathrm{CPW}$ of the phase shifter to the coplanar strip feeding line of the dipole antenna. The Si chip, consisting in an antenna array of two elements in an area of $6 \times 7 \mathrm{~mm}^{2}$, was successfully fabricated and assembled to a customized PCB with an SMA connector for testing (Fig. 19(b)).

The first measurements of the beam steering concept demonstrate good return loss performance with small deviation versus simulations $[22,23]$. Typical antenna radiation pattern behavior was measured and active beam steering measurements are currently ongoing.

\section{TUNABLERF-MEMS FILTERS - FILTERING}

\section{A) Bandpass filter}

A compact and simple controllable tunable bandpass filter has been fabricated based on a lumped-distributed transmission line filter concept [24] to select discretely the specific center frequency (from 20 to $23 \mathrm{GHz}$ ) with an independent controllable channel bandwidth (of approximately 0.5-1 GHz).

The electrical length of the filter corresponds approximately to $\lambda / 2$ at the maximum center frequency of the tuning range in a slow-wave structure (meaning the CPW including the MEMS loaded capacitors in the up-state). The filter combines two kinds of MEMS devices shown in the SEM image of the filter partly represented in Fig. 20. The first kind of devices, the shunt digital capacitors, are used to digitally tune from one frequency band implemented by using the concept of DMTL lines [10]. Changing the state of these capacitors discretely modify the phase velocity and electrical length of the resonator and thus, the resonant frequency. The second part of the system is implemented with MEMS interdigitated capacitors in series configuration 


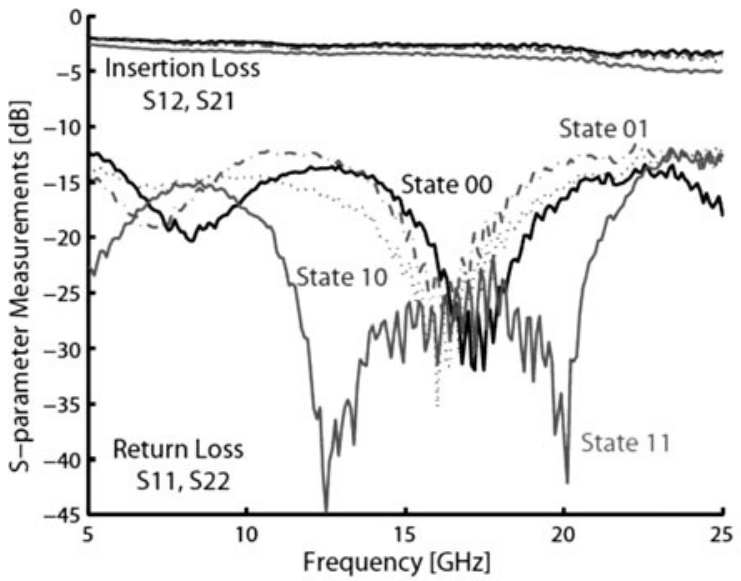

(a)

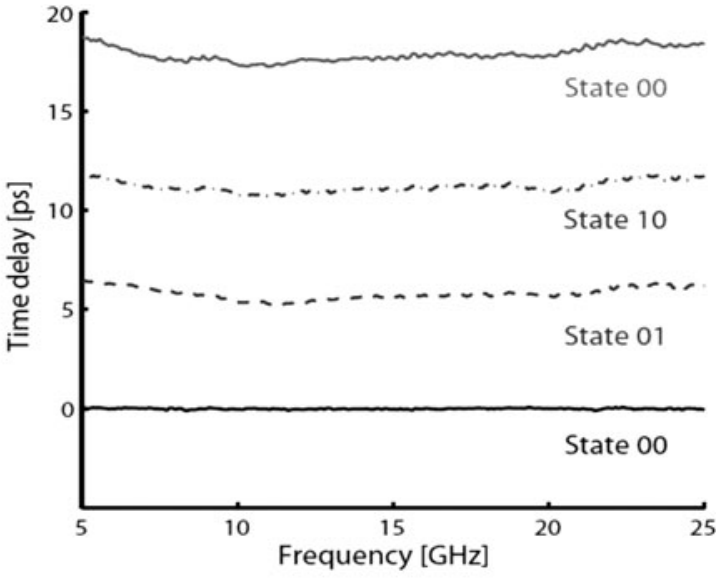

(b)

Fig. 17. Two-bit IB-DMTL phase shifter with an enhanced optimization method to improve the matching at $17 \mathrm{GHz}$. (a) $S$-parameter measurements and (b) constant time delay states at different actuation bits.
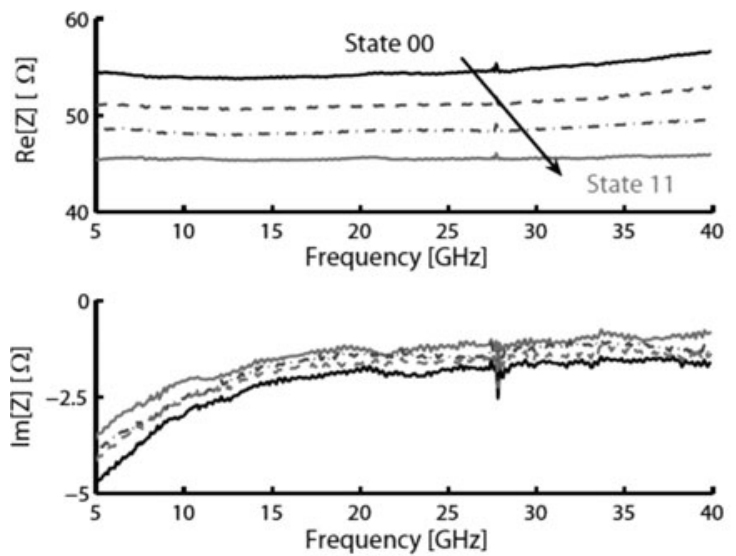

(a)

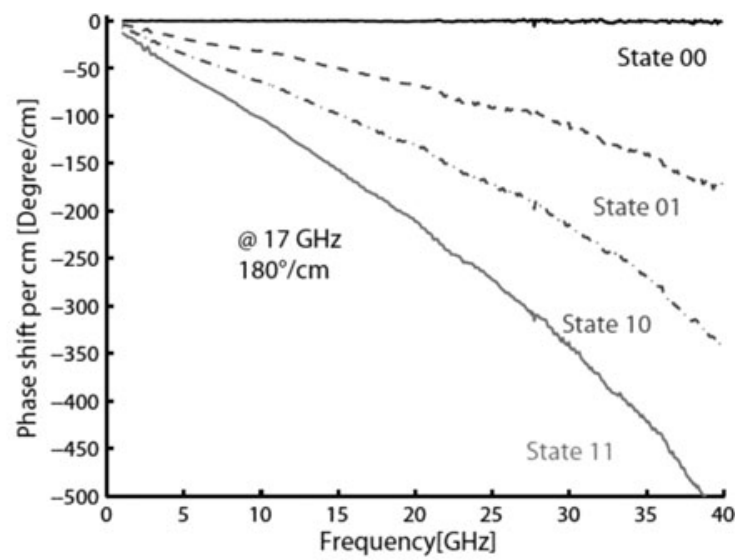

(b)

Fig. 18. Two-bit IB-DMTL frequency optimized phase shifter; (a) the real and imaginary impedance and (b) constant time delay states at different actuation bits.

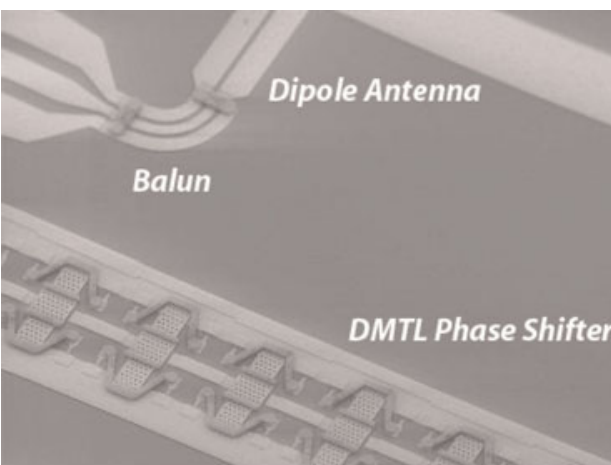

(a)

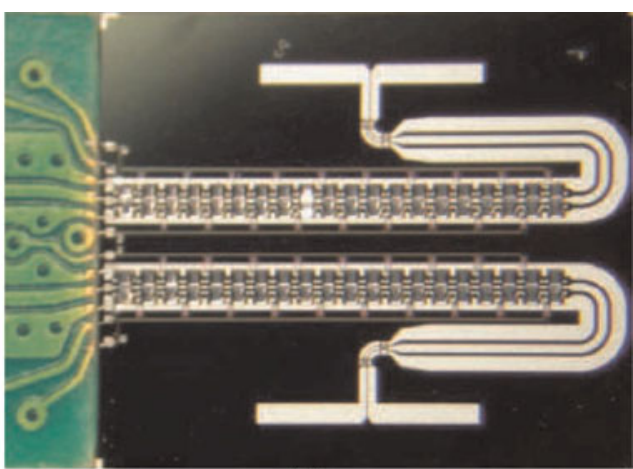

(b)

Fig. 19. (a) SEM image of the fabricated entire system for applications in antenna beam steering and (b) optical image of the antenna array integration with the PCB showing the wire bonding connections.

(series-analog capacitor). They work in analog continuous mode and its main function is to continuously tune the bandwidth of the bandpass filter by modifying the value of the coupling series-analog capacitor placed at both the input and the output of the filter. In addition, they can be used to fine de-tune the filter center frequency by an extra correcting element originally placed at the center of the filter and first introduced by the authors in [24].

$S$-parameter measurements were taken from 15 to $25 \mathrm{GHz}$ with a center frequency in $f_{\mathrm{o}}=21.5 \mathrm{GHz}$. Actuation of the analog and digital series and shunt MEMS capacitors was accomplished with voltages from 15 to $40 \mathrm{~V}$. 


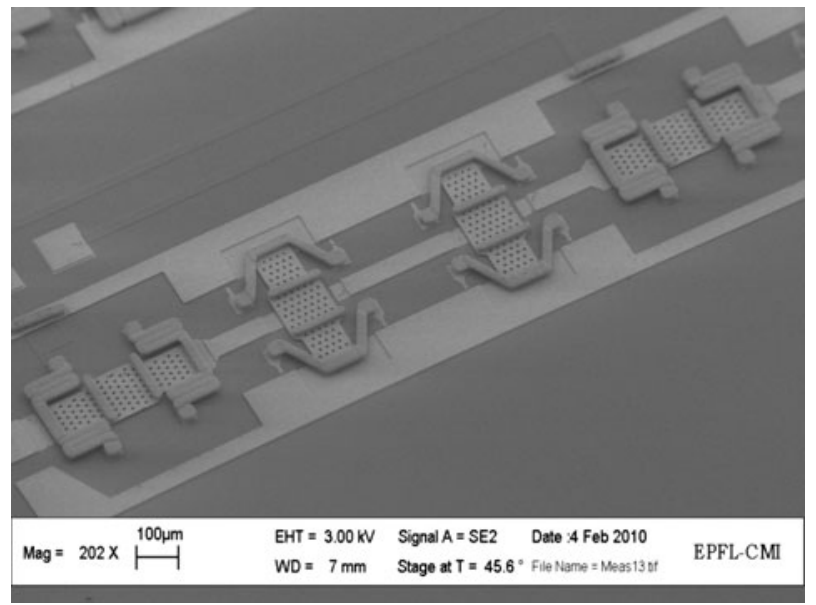

Fig. 2o. SEM images of the fabricated tunable bandpass filter with parallel and series MEMS devices.

The center frequency of the bandpass filter shifts from 20.45 to $22.6 \mathrm{GHz}$ which corresponds to a $9.53 \% f_{\mathrm{o}}$ tuning with relative stable fractional bandwidth $(15.1 \pm 1.9 \%)$ and minimum variation of the insertion loss (4.05 \pm $0.25 \mathrm{~dB}$ ) across the complete tuning range (Fig. 21(a)). Tuning of the bandwidth is also demonstrated in a constant center frequency, thanks to action of the correcting element [24]. A $13.6 \%$ of bandwidth tuning is achieved at $20.5 \mathrm{GHz}$ center frequency with a $1 \mathrm{~dB}$ fractional bandwidth varying from 17 to $20 \%$. It is the first time that such independent center frequency and bandwidth tuning is demonstrated for filters working in the K-band of frequencies. Similar performance can be found in the literature but for filters working in the lower UHF frequency bands (from $300 \mathrm{MHz}$ to $1.4 \mathrm{GHz}$ ) [25].

\section{B) Band-stop filter}

A wideband high-frequency tunable band-stop filter working in the $\mathrm{Ku}$ - to $\mathrm{Ka}$-band frequencies was originally devised $[14,26]$. The filter is based on a miniature single-MEM device combining a central capacitive MEM switch loaded in a CPW line and suspension arms designed as multiple inductive meanders with voltage-controlled coupling to the substrate (Fig. 22).

The meander-type anchoring of the suspended structure has been specifically designed to modify the inductance of the LC tank to resonate at the frequency of interest and producing a considerable notch response. The main tuning capability is achieved, thanks to the variable capacitance between the suspended metal membrane and the RF signal bottom electrode via an electrostatic actuation. The tuning range can be increased by reducing the stiffness of the anchors, thus creating a zipping effect after the pull-in, avoiding a perfect contact of the membrane at pull-in. Due to the fabrication-induced stress and the low stiffness of the bridge at zero bias, the central part of the bridge slightly touches the bottom electrode, enabling an enlarged stable tuning region before pull-in (capacitance ratio in the stable region, $C r=1.5$ ) and a zipping effect after the pull-in. The tuning behavior is well controlled and the capacitance smoothly changes in a repeatable way and in a continuous and quasilinear fashion, until an improved full contact of both electrodes.

In addition, the intrinsic inductance of the meanders over the slot (between the RF signal and the ground planes of the CPW) plays a significant role in the device operation as their coupling to the substrate additionally tune the capacitive component of the device due to the bending of the metal meander anchors during electrostatic actuation (Fig. 22(b)).

The fabricated device has an air gap of $\sim_{3} \mu \mathrm{m}$. The width and length of the meanders are 10 and $135 \mu \mathrm{m}$, respectively, and the capacitive actuation area is $135 \mu \mathrm{m} \times 80 \mu \mathrm{m}$. The tunable band-stop filter has been successfully characterized at room temperature and under high vacuum $\left(10^{-5} \mathrm{mbar}\right)$. High tuning range of the filter center frequency of over $55 \%$ is achieved. The center frequency tunes from 16.7 to $37 \mathrm{GHz}$ (Fig. 23). A linear capacitive tuning is achieved from o to $27.5 \mathrm{~V}$ which corresponds to a continuous linear frequency tuning of $20 \%$. The two center frequency tuning mechanisms are visible in Fig. 23. First, the MEMS capacitive tuning and, second, the zipping effect of the inductive arm coupling capacitance can be seen in this figure.

The insertion loss in the lower pass-band is better than $0.25 \mathrm{~dB}$ and the rejection level is around $20 \mathrm{~dB}$ for all the

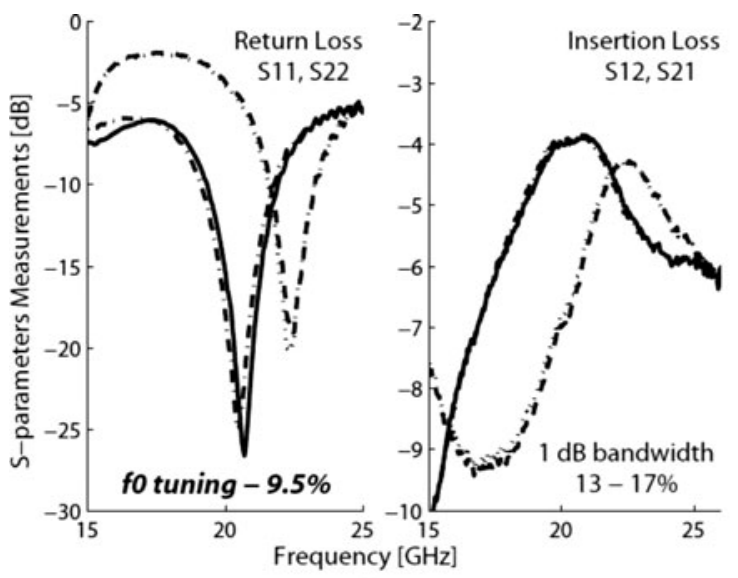

(a)

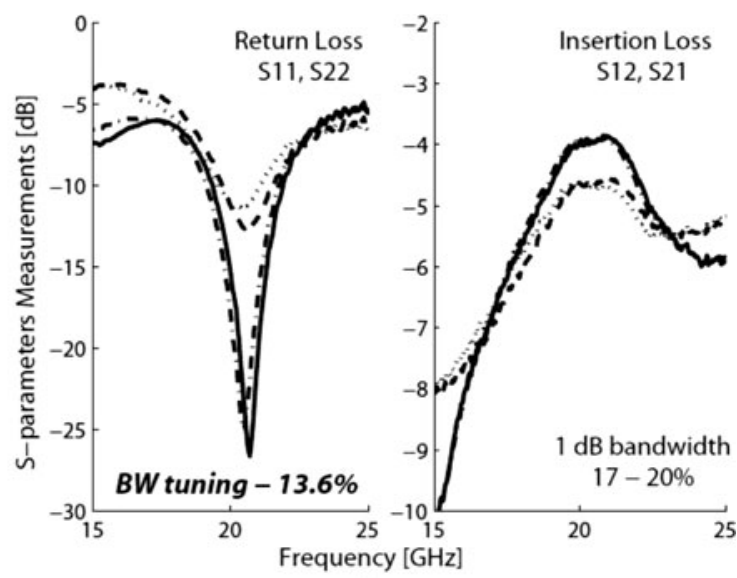

(b)

Fig. 21. S-parameter measurements of a distributed-lumped bandpass filter with independent (a) center frequency tuning from 20.5 to $22.5 \mathrm{GHz}$ ( $9.5 \%$ ) and (b) a relative bandwidth tuning of $13.6 \%$. 


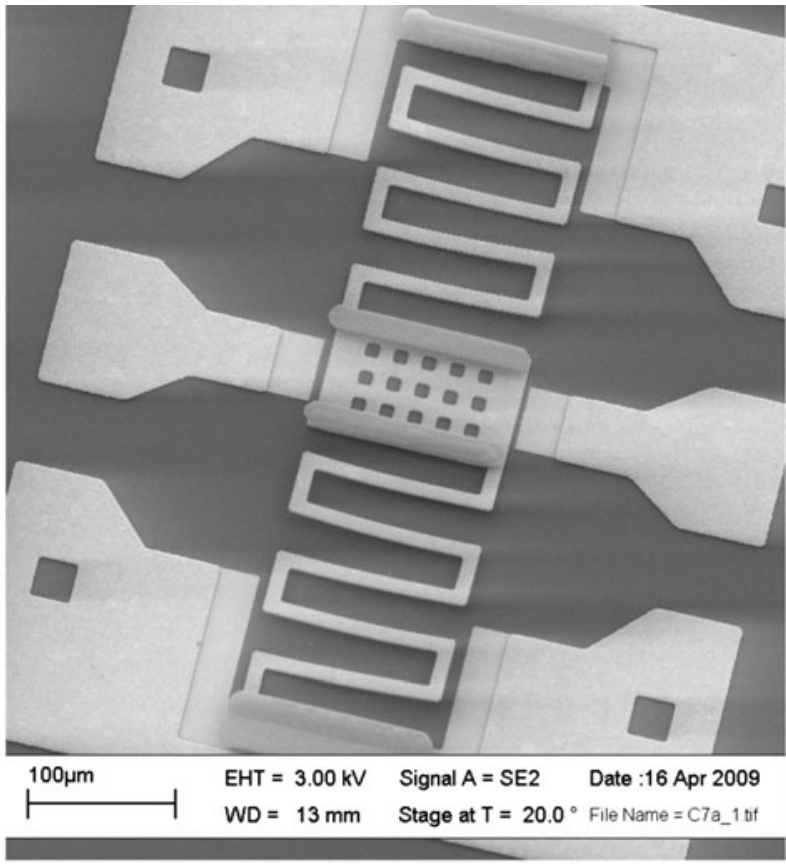

(a)

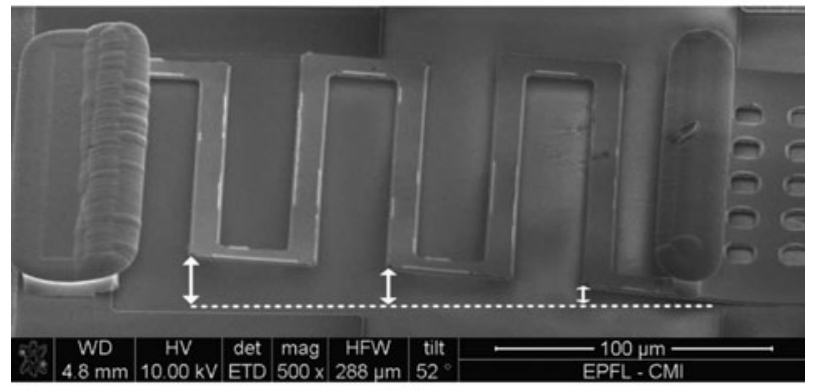

(b)

Fig. 22. SEM images of (a) the fabricated band-stop filter device and (b) the meandering arm from the filter showing the zipping effect of the anchor.

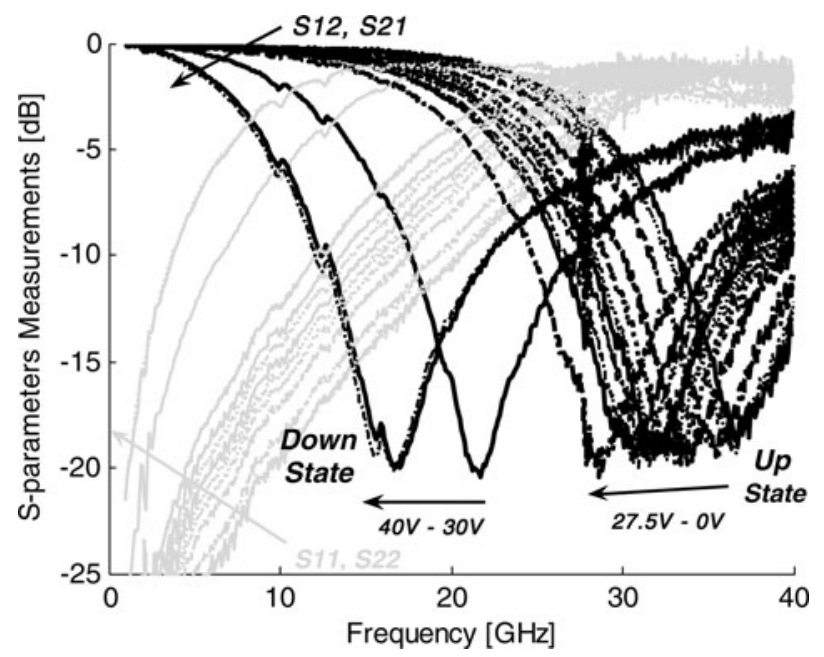

Fig. 23. S-parameters of the band-stop filter showing an outstanding tuning range of over $55 \%$.

rejection bands. A constant fractional bandwidth of 3.3 and 5.7 $\mathrm{GHz}$ has been measured at 50 and $75 \%$ of the rejection level of the filter $(-3$ and $-6 \mathrm{~dB})$ which corresponds to a relative bandwidth of $10 \%$. The temperature dependence is
4.5 $\mathrm{MHz} /{ }^{\circ} \mathrm{C}$ for the temperature range from 20 to $100^{\circ} \mathrm{C}$ and gets around the double for the extended range $(-100$ to $\left.100^{\circ} \mathrm{C}\right)$. The fabricated device is very robust and show highly reproducible characteristics at wafer level; the filter characteristics in air and vacuum are quasi-identical and stay stable over 3 month of wafer storage in ambient conditions.

\section{CONCLUSIONS}

This paper presents a detailed investigation of RF-MEMS capacitive switches using AlN as dielectric and $\mathrm{Ni}$ as the structural material in phase shifting and filtering functions. Materials and design optimization have allowed the reduction of insertion losses and dissipation in miniaturized systems with novel functionalities. The presented RF-MEMS technology is not only a solution for improved RF performance in a wideband $(5-40 \mathrm{GHz})$ but also shows high robustness and reliability.

\section{ACKNDWLEDGEMENT}

The authors would like to thank T. Lisec from IsiT Fraunhofer, Germany for the fabrication support and E. Buitrago for language revision. This work was partially supported by the $\mathrm{FP}_{7}$ Integrated project e-CUBES, e-BRAINS, and by the Thales Company, France.

\section{REFERENCES}

[1] Rebeiz, G.M.: RF MEMS: Theory, Design and Technology. Wiley-Interscience, New York, 2003.

[2] Goldsmith, C.L.; Zhimin, Y.; Eshelman, S.; Denniston, D.: Performance of low-loss RF MEMS capacitive switches. IEEE Microw. Guided Wave Lett., 8 (8) (1998), 269-271.

[3] Peroulis, D.; Pacheco, S.P.; Sarabandi, K.; Katehi, L.P.B.: Electromechanical considerations in developing low-voltage RF MEMS switches. IEEE Trans. Microw. Theory Tech., 51 (1) (2003), 259-270.

[4] Park, J.Y.; Kim, G.H.; Chung, K.W.; Bu, J.U.: Fully integrated micromachined capacitive switches for RF applications, in Proc. IEEE MTT-S Int. Microw. Symp. Dig., vol. 1, June 2000, 283-286.

[5] Blondy, P. et al.: Dielectric less capacitive MEMS switches, in Proc. IEEE MTT-S Int. Microw. Symp. Dig., vol. 2, June 2004, 573-576.

[6] Ziaei, A.; Dean, T.; Mancuso, Y.: Lifetime characterization of capacitive power RF MEMS switches, in Proc. 35th Eur. Microw. Conf., vol. 3, October 2005, 4.

[7] Fernández-Bolaños, M.; Tsamados, D.; Dainesi, P.; Ionescu, A.M.: Reliability of RF MEMS capacitive switches and distributed MEMS phase shifters using AlN dielectric, in Proc. IEEE 22nd Int. Conf. Micro Electro Mech. Syst., January 2009, 638-641.

[8] Muldavin, J.B.; Rebeiz, G.M.: High-isolation inductively-tuned $\mathrm{X}$-band MEMS shunt switches, in Proc. IEEE MTT-S Int. Microw. Symp. Dig., vol. 1, June 2000, 169-172.

[9] Hayden, J.S.; Rebeiz, G.M.: Low-loss cascadable MEMS distributed X-band phase shifters. IEEE Microw. Guided Wave Lett., 10 (4) (2000), 142-144. 
[10] Fernández-Bolaños, M.; Lisec, T.; Dainesi, P.; Ionescu, A.M.: Thermally stable distributed MEMS phase shifter for airborne and space applications, in Proc. 38th Eur. Microw. Conf., October 2008, 100-103.

[11] Perruisseau-Carrier, J.; Fritschi, R.; Crespo-Valero, P.; Skrivervik, A.K.: Modeling of periodic distributed MEMS-application to the design of variable true time delay lines. IEEE Trans. Microw. Theory Tech., 54 (1) (2006), 383-392.

[12] Goldsmith, C.; Maciel, J.; McKillop, J.: Demonstrating reliability. IEEE Microw. Mag., 8 (2007), 56-60.

[13] Lakshminarayanan, B.; Mercier, D.; Rebeiz, G.M.: High-reliability miniature RF-MEMS switched capacitors. IEEE Trans. Microw. Theory Tech., 56 (4) (2008), 971-981.

[14] Fernández-Bolaños, M.; Lisec, T.; Dehollain, C.; Tsamados, D.; Nicole, P.; Ionescu, A.M.: Highly tunable band-stop filters based on AlN RF MEMS capacitive switches with inductive arms and zipping capacitive coupling, in Int. Electron Device Meeting, IEDM 2009, December 2009, 805-808.

[15] Fernández-Bolaños, M.; Tsamados, D.; Ionescu, A.M.: RF performance and lifetime study of MEMS capacitive switches using AlN as dielectric compared to $\mathrm{Si}_{3} \mathrm{~N}_{4}$. J. Microelectromech. Syst, (2010).

[16] Simons, R.: Coplanar Waveguide Circuits, Components and Systems. John Wiley and Sons, Inc., New York, 2001.

[17] Vinoy, K.J.; Varadan, V.K.: Design of reconfigurable fractal antennas and RF-MEMS for space-based systems. Smart Mater. Struct., 10 (2001), 1211-1223.

[18] Barker, S.; Rebeiz, G.M.: Distributed MEMS true-time delay phase shifter and wide-band switches. IEEE Trans. Microw. Theory Tech., 46 (11) (1998), 1881-1890.

[19] Hung, J.-J.; Dussopt, L.; Rebeiz, G.M.: Distributed 2- and 3-bit W-band MEMS phase shifters on glass substrates. IEEE Trans. Microw. Theory Tech., 52 (2) (2004), 600-606.

[20] Perruisseau-Carrier, J.; Fritschi, R.; Skrivervik, A.K.: Design of enhanced multi-bit distributed MEMS variable true-time delay lines, in Research in Microelectron. Electron., $\mathrm{PhD}$, vol. 1, July 2005, 189-192.

[21] Fernández-Bolaños, M.; Perruisseau-Carrier, J.; Dainesi, P.; Ionescu, A.M.: RF MEMS capacitive switch on semi-suspended CPW using low-loss high-resistivity silicon substrate. J. Microelectron. Eng., 85 (5-6) (2008), 1039-1042.

[22] Fernández-Bolaños, M. et al.: Dipole antenna and distributed MEMS phase shifter fully integrated in a single wafer process for beam steering applications. J. Microelectron. Eng., 87 (2010), 1290-1293.

[23] Vasylchenko, A.; Fernández-Bolaños, M.; Brebels, S.; De Raedt, W.; Vandenbosch, G.A.E.: Conformal phased array for a miniature wireless sensor node, in IEEE 2oth Int. Conf. on Applied Electromagnetics and Comm., September 2010 (invited).

[24] Fernánez-Bolaños, M.; Dehollain, C.; Ayoz, S.; Nicole, P.; Ionescu, A.M.: Center frequency and bandwidth tunable bandpass filter based on RF MEMS (10 GHz- 14 GHz), in Proc. 39th Eur. Microw. Conf., September 2009, 1708-1711.

[25] Carey-Smith, B.E.; Warr, P.A.; Beach, M.A.; Nesimoglu, T.: Wide tuning-range planar filters using lumped-distributed coupled resonators. IEEE Trans. Microw. Theory Tech., 53 (2) (2005), $777-785$.

[26] Fernández-Bolaños, M.; Dehollain, C.; Nicole, P.; Ionescu, A.M.: Tunable band-stop filter based on single RF MEMS capacitive shunt switch with meander arm inductance. J. Solid-State Electronic, 54 (9) (2010), 1033-1040.

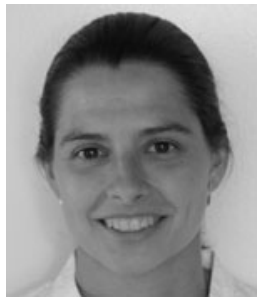

Montserrat Fernández-Bolaños Badía received her M.Sc. degree in Telecommunication Engineering from the University of Seville, Spain, in 2005. She received her Ph.D. degree in Microsystems and Microelectronic from Ecole Polytechnique Fédérale de Lausanne (EPFL) in 2010. Since August 2010, she has been working as a scientific collaborator in the Nanoelectronic device laboratory (NANOLAB) at EPFL. The focus of her research is in the field of RF-MEMS switches, phase shifter, and tunable filters for airborne and space applications. Her present research interests include the open challenges of RF-MEMS devices such as the reliability, the packaging, and their $3 \mathrm{D}$ integration with RF ICs.

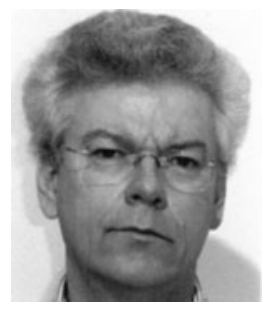

Pierre Nicole is the R\&D project manager at THALES Airborne Systems. He has more than 30 years of experience in the fields of Optoelectronics and Microwave Functions and Systems R\&D. He received his eng. degree from INPG (ENSERG) in 1980 and joined SAT to participate in the first projects in France on optical links for telecommunications. In 1984, he then joined the Technical Directorate of Dassault Electronique, where he continued works on digital and microwave optical links for harsh aeronautical systems. He then contributed to the development of the new field of microwave wireless sensor networks in 1994, where he developed demonstrators of wireless sensors embedded in concrete, and patented different issues about "Abandoned Sensors" remotely or self-energized through microwave, optical, and acoustical waves. He holds various patents in each field. His current R\&D activities are related to millimeter-wave optical links, microwave MEMS functions and applications, wireless sensors, smart antennas, and autonomous microsystems.

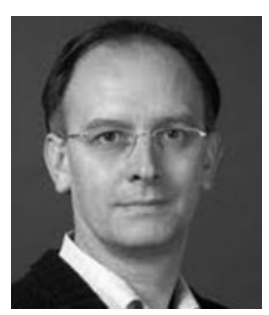

Adrian Mihai Ionescu is an Associate Professor at the Swiss Federal Institute of Technology, Lausanne, Switzerland. He received the B.S./M.S. and Ph.D. degrees from the Polytechnic Institute of Bucharest, Romania and the National Polytechnic Institute of Grenoble, France, in 1989 and 1997, respectively. He has held staff and/or visiting positions at LETI-CEA, Grenoble, France, LPCS-ENSERG, Grenoble, France, and Stanford University, USA, during 1998 and 1999. His research interests focus on micro- and nano-electronic devices aimed at Integrated Circuit design especially process development, modeling, and electrical characterization. Dr. Ionescu has published more than 250 articles in international journals and conferences. He received three best paper awards in international conferences and the Annual Award of the Technical Section of the Romanian Academy (of Sciences) in 1994. He is director of the Laboratory of Micro/Nanoelectronic Devices (NANOLAB) and head of the Doctoral School in Microsystems and Microelectronics of EPFL. 